\title{
Flame Spread in Laminar Mixing Layers: The Triple Flame
}

\author{
P. N. KIONI, B. ROGG,* AND K. N. C. BRAY
}

\begin{abstract}
A. LIÑ̂́N
In the present paper we investigate flame spread in laminar mixing layers both experimentally and numerically. First, a burner has been designed and built such that stationary triple flames can be stabilised in a coflowing stream with well defined linear concentration gradients and well defined uniform flow velocity at the inlet to the combustion chamber. The burner itself as well as first experimental results obtained with it are presented. Second, a theoretical model is formulated for analysis of triple flames in a strained mixing laycr generated by directing a fuel stream and an oxidizer stream towards each other. Here attention is focused on the stagnation region where by means of a similarity formulation the three-dimensional flow can be described by only two spatial coordinates. To solve the governing equations for the limiting case in which a thermal-diffusional model results, a numerical solution procedure based on self-adaptive mesh refinement is developed. For the thermal-diffusional model, the structure of the triple flame and its propagation velocity are obtained by solving numerically the governing similarity equations for a wide range of strain rates.
\end{abstract}

\section{INTRODUCTION}

Laminar flames in turbulent flows are subjected to strain and develop curvature as consequences of the turbulent velocity fluctuations. Effects of both influences, combined with effects of differential diffusion of heat and reactants, cause the inner structure of the flames to respond, thereby leading in some cases to extinction. These phenomena have important implications on the modeling of turbulent reacting flows in the laminar-flamelet regime. To enhance their understanding, a detailed knowledge of the laminar-flame structure as a function of various parameters, such as the rate of strain, the radius of curvature, the type of fuel, or the equivalence ratio, as well as its response to variations of these parameters is required. For instance, in turbulent diffusion flames on the molecular level, socalled "triple flames" may form when after local flamelet extinction due to excessive straining the turbulence intensity decreases to values sufficiently low for reignition to take place. It is the purpose of the present article to investigate such laminar triple flames in turbulent flows.

Triple flames can occur in a variety of geometries. Figure 1 shows a photograph of a stationary triple flame stabilized in upwardly coflowing streams of fuel and oxidizer using the burner described in subsection 2.1 of the present paper. The fresh mixture ahead of the flame is nonuniform in that the fuel and oxidizer concentrations vary linearly from pure fuel at the left side to pure oxidizer at the right; the flow velocity ahead of the flame is uniform. ${ }^{1}$ The flame structure is composed of (i) an upward bending fuel-rich premixed flame on the left-hand side, (ii) an upward bending fuel-lean premixed flame on the right-hand side, and (iii) a thin, long, trailing, streamwise 


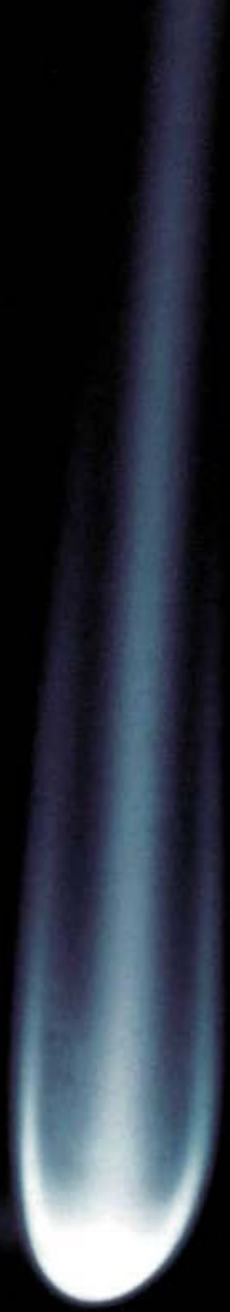

Fig. 1. Typical photograph of a stationary triple flame in a coflowing stream stabilized in the burner described herein. Details can be found in subsections 2.1 and 2.2. 
diffusion flame between the two premixed flames in which the excess fuel and oxidizer not consumed in the rich and lean premixed flame, respectively, burn. The region, where the two premixed flames and the diffusion flame merge, is referred to as "triple-point region" or, alternatively, as "triple point." Therefore, the flame itself is referred to as a "triple flame." It should be noted here that triple flames were observed earlier by Phillips [1], who also presented photographic evidence.

Although a number of theoretical, experimental, and computational studies have been devoted to steadily burning premixed, partially premixed, and non-premixed laminar counterflow flames, only a few studies have addressed the propagation of laminar flames in mixing layers. Liñán and Crespo [2] used asymptotic methods to analyze the transient mixing in a boundary layer of two initially separated coflowing streams of fuel and oxidizer, and demonstrated the existence of multiple burning regimes. Also using theoretical methods, Dold [3-6], Buckmaster and Matalon [7], and Wichman [8] carried out analyses of various limiting combustion regimes based on somewhat more general governing equations that were not subjected to the boundary-layer approximation. A numerical analysis of a laminar coflow diffusion flame, established in channels at the trailing edge of a splitter plate separating coflowing streams of fuel and oxidizer, was performed by Ramanujam and Tien [9]. With notable exceptions $[5,6]$, in the above analyses effects of strain were not taken into account.

The purpose of the present article is twofold. First, a triple-flame burner is presented which has been designed and built such that stationary triple flames can be stabilized in a coflowing stream with well defined linear concentration gradients and well-defined uniform velocity profile at the inlet to the combustion chamber. The burner itself as well as first experimental results obtained with it are presented.

Second, a theoretical model is formulated for analysis of steadily propagating triple flames in a strained mixing layer generated by counterflowing streams of fuel and oxidizer. In particular, for the latter geometry a general similarity formulation is derived which describes the complex three-dimensional process of flame spread by only two spatial coordinates. To solve the nonlinear governing similarity equations, a numerical solution procedure based on selfadaptive mesh refinement is developed. For the limiting case, in which a thermal-diffusional model results, the numerical procedure is used to calculate the structure of the flame and its propagation velocity ${ }^{2}$ for a wide range of strain rates.

\section{EXPERIMENTAL}

\subsection{Experimental Setup}

Figures $2 \mathrm{a}-2 \mathrm{c}$ are sectional views highlighting the important aspects of the burner. The triple flame is stabilised in the mixing layer as illustrated in Figs. $2 a$ and $2 b$. Figure $2 b$ shows a section of the burner viewed from the front: in this view the two premixed-flame wings and the trailing diffusion-flame wing of the triple flame are clearly visible. It is from this view that the photograph shown in Fig. 1 has been taken. Figure $2 b$ shows a burner section from the side. From this view only the sides of the front premixed-flame wing and of the upper part of the diffusion-flame wing are visible; the lower part of the diffusion-flame wing, the rear premixed-flame wing and the triple-point region are "hidden" behind the front premixed-flame wing. At the inlet of the combustion chamber, in the plane of the triple flame, the composition varies linearly from one side to the other side; the composition is constant in the direction perpendicular to the triple flame. The velocity at the inlet to the combustion chamber is uniform. The triple flame is stabilized in the combustion chamber by a negative velocity gradient in the streamwise direction, which is the result of the diverging chamber walls; see Fig. 2b. The angle of the divergence of the walls is adjustable. The experimental rig is designed such that a wide range of inlet concentration gradients can be realized for a wide range of uniform inlet velocities. Thus, the rig 

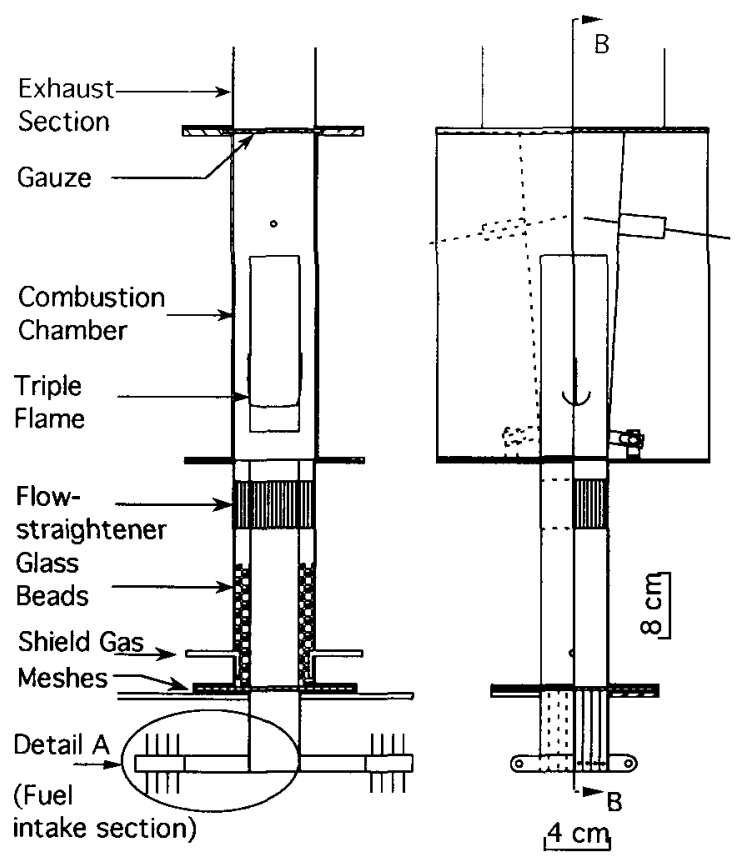

(a)

SECTION B-B

(b)

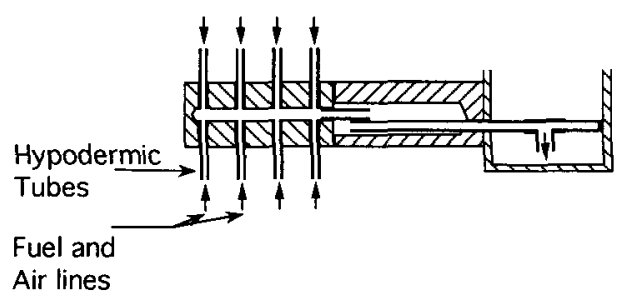

(c)

Detail A: Flow of the Fuel-Air Mixture Through the Mixing Block

Fig. 2. Sectional views of the burner: (a) side view; (b) front view; (c) view of the mixing block. A detailed discussion of this figure is given in subsection 2.1.

allows stationary triple flame of different width at the leading edge of the flame to be generated.

The smooth linear concentration profiles upstream of the triple flame are obtained from stepwise uniform profiles at the outlet of the compartments shown at the bottom of Fig. 2b; at the outlet of each compartment the composition is uniform. The stepwise concentration profiles decay in the mixing region towards smooth linear profiles at the entrance to the combustion chamber.

Different fuel mass fraction gradients at the inlet to the combustion chamber are obtained by varying the fuel-concentration gradient of the stepwise uniform profile. This is achieved by suitably adjusting the composition of the mixtures introduced into the burner through the different compartments. The adjustment of the composition in an individual compartment is accomplished by using a system of hypodermic tubes through which air and fuel, originating from different manifolds, pass before entering the compartments. Metering of the gases from the different manifolds to each compartment is based on the Hagen-Poiseuille relationship for laminar pipe flow. Thus the mass flow rates are determined from the readings of the gas pressure in the manifolds.

Figure $2 \mathrm{c}$ shows how mixing of fuel and oxidizer is achieved in a mixing block such that the composition is uniform when the flow enters the respective compartment. Fuel and oxidizer arrive from opposite sides at the mixing block through a certain number of hypodermic tubes. In each of these tubes the flow is that of either pure fuel or pure oxidizer, with a flow rate such that at the exit from the mixing block the desired composition is achieved. In each mixing block, impingement on each other of the small jets issued by the hypodermic tubes leads to a first mixing of fuel and oxidizer. Further mixing of fuel and oxidizer, which, for the individual compartments then results in a homogeneous mixture, is achieved by directing the gas flow through an S-shaped, circuitous path as shown in Fig. 2c.

\subsection{Experimental Results and Discussion}

The experimental results presented here were obtained using $45 \%$ (based on volume) methane in nitrogen. Figure 1 is a photograph of a typical triple flame obtained in the burner described above, showing the lean premixed flame on the right-hand side, the rich premixed flame on the left-hand side, and the trailing diffusion flame in the middle. Note that in Fig. 1 , further downstream of the leading edge of the triple flame, the premixed wings of the flame are parallel. This was observed for most of the triple flames, but for some flames there was a slight downstream convergence of the tails of the premixed wings.

Figure $3 \mathrm{a}$ shows the variation of the triple flame width, $W$, defined as the maximum sepa- 


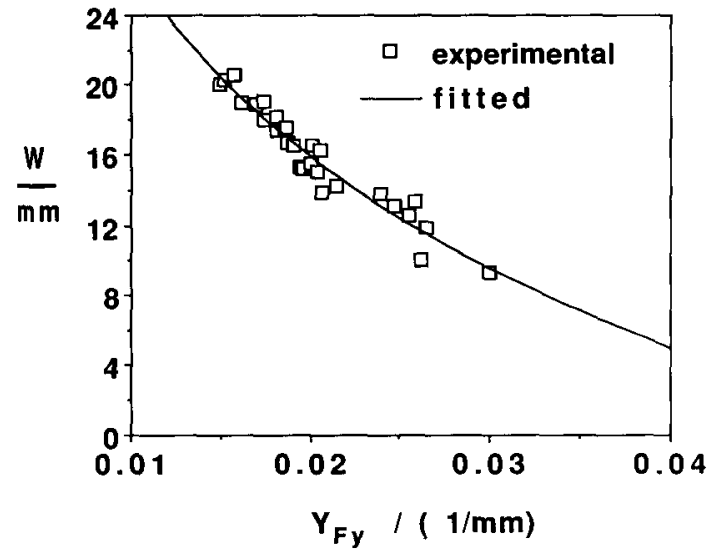

(a)

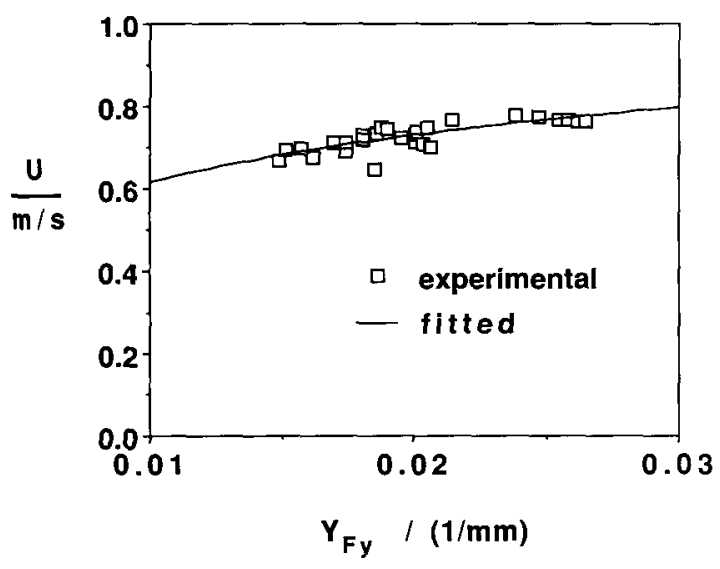

(b)

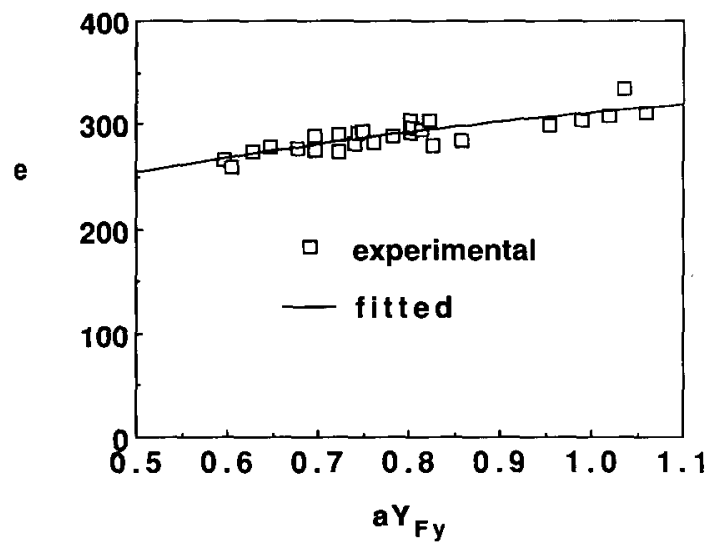

(c)

Fig. 3. Experimental results: width $W$ of the triple flame as a function of the inlet fuel mass fraction gradient (a), velocity $U$ of the triple flame as a function of the inlet fuel mass fraction gradient (b), and nondimensional parameter $e$ as a function of the nondimensional parameter $a Y_{F y}$ (c); for details, see subsection 2.2 .

\section{fuel, $v_{\text {fuel }}$ \\ $1+1+11$

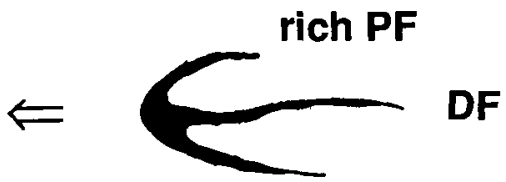 \\ lean PF
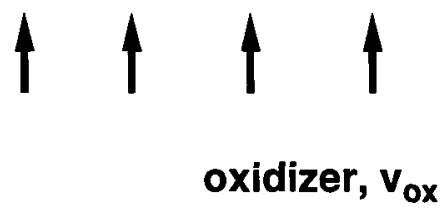

Fig. 4. Schematic of a triple flame propagating steadily from right to left in a strained mixing layer generated by directing a stream of fuel (from the top with velocity $v_{\text {fuel }}$ ) and oxidizer (from the bottom with velocity $v_{\mathrm{ox}}$ ) towards each other; $\mathrm{PF}=$ premixed flame, $\mathrm{DF}=$ diffusion flame.

ration distance of the premixed wings of the flame, with the fuel mass fraction gradient at the inlet to the combustion chamber, $Y_{F y}$.

Figure $3 \mathrm{~b}$ shows the variation of the velocity $U$ with the inlet fuel mass fraction gradient $Y_{F y}$. Here $U$ is defined as the mean flow velocity at the location of the flame; it has been calculated by dividing the volumetric flow rate by the cross-sectional area of the burner at the height of the leading edge of the flame. The velocities thus obtained are much higher than the adiabatic laminar burning velocity for the corresponding premixed stoichiometric fuel-air mixture. This observation is consistent with observations reported by Phillips [1].

Figure $3 \mathrm{c}$ shows the nondimensional parameter

$e=\frac{a Y_{F y}}{b / W}$

as a function of the nondimensional inlet mass fraction gradient, $a Y_{F y}$, of the fuel. Here, in addition to the quantities $Y_{F y}$ and $W$ defined above, $a$ is the width of the combustion chamber at the inlet and $b$ is its width at the leading edge of the triple flame, that is, at the height of the triple point. Note that $e$ can be inter- 
preted as the ratio of the nondimensional width of the flame to the nondimensional width of the mixing layer. It is seen that $e$ varies only slightly with $a Y_{F y}$.

\section{MODELING AND SIMULATION}

\subsection{The Governing Equations in Similarity Form}

As independent variables the time $t$ and rectangular cartesian space coordinates $x, y$, and $z$ are adopted. Although herein we are interested only in steady phenomena and, therefore, will adopt time-independent boundary conditions, the time-dependent terms are retained in the governing equations to allow a transient approach to the steady solution of the problem. In seeking a similarity formulation, we assume that for all quantities, except the pressure $p$ and the $z$ velocity component $w$, spatial variations occur only with respect to the $x$ and $y$ direction. Specifically, the velocity field $\boldsymbol{v} \equiv(u, v, w)$ is assumed to be of the form

$u=u(t, x, y), \quad v=v(t, x, y)$,

$w=z A(t, x, y)$,

where $A=A(t, x, y)$ is the variable strainrate; the pressure $p$ is taken as

$p=p_{O}+p^{\prime}(t, x, y)-A_{O}^{2} \rho_{O}\left(\frac{y^{2}}{2}+\frac{z^{2}}{2}\right)$,

where the subscript $O$ identifies the constant values of the respective quantities prevailing at the oxidizer boundary. The functional dependence of temperature and mass fractions is

$T=T(t, x, y) \quad$ and $\quad Y_{i}=Y_{i}(t, x, y)$,

respectively, $i=1, \ldots, N$, where $N$ denotes the number of chemical species in the system. We consider low-Mach-number flow of ideal-gas mixtures. Effects of body forces, bulk viscosity, viscous dissipation, and thermal diffusion are neglected; ordinary diffusion is assumed to obey Fick's law with suitably defined diffusion coefficients $D_{i}$ to be specified below. Furthermore, pressure-gradient diffusion and effects of pressure variations on temperature are neglected. Thus, in terms of the accumulativeconvective-diffusive transport operator

$$
\begin{aligned}
L(\phi ; \Gamma) \equiv & \frac{\partial(\rho \phi)}{\partial t}+\frac{\partial(\rho u \phi)}{\partial x}+\frac{\partial(\rho v \phi)}{\partial y} \\
& +\rho \phi A-\frac{\partial}{\partial x}\left(\Gamma \frac{\partial \phi}{\partial x}\right)-\frac{\partial}{\partial y}\left(\Gamma \frac{\partial \phi}{\partial y}\right) \\
= & \rho \frac{\partial \phi}{\partial t}+\rho u \frac{\partial \phi}{\partial x}+\rho v \frac{\partial \phi}{\partial y} \\
& -\frac{\partial}{\partial x}\left(\Gamma \frac{\partial \phi}{\partial x}\right)-\frac{\partial}{\partial y}\left(\Gamma \frac{\partial \phi}{\partial y}\right)
\end{aligned}
$$

where $\phi$ denotes any of the quantities $\rho, u, v$, $A, T$, or $Y_{i}$, the governing equations can be written as

$$
\begin{aligned}
& L(1 ; 1)=0, \\
& L(u ; \mu)=-\frac{\partial p^{\prime}}{\partial x}+U, \\
& L(v ; \mu)=-\frac{\partial p^{\prime}}{\partial y}+\rho_{O} A_{O}^{2} y+V, \\
& L(A ; \mu)=\rho_{O} A_{O}^{2}-\rho A^{2}, \\
& c_{p} L\left(T ; \lambda / c_{p}\right)=(\nabla T) \cdot \sum_{i=1}^{N} c_{p i}\left(\rho D_{i} \nabla Y_{i}\right) \\
&-\sum_{i=1}^{N} h_{i} w_{i}+H, \\
& L\left(Y_{i} ; \rho D_{i}\right)= w_{i},
\end{aligned}
$$

$i=1, \ldots, N$. In addition to the quantities already defined, in Eqs. 6-10 $\mu$ denotes the dynamic viscosity of the mixture, $\lambda$ its thermal conductivity, and $c_{p}$ its constant-pressure specific heat capacity; $c_{p i}, h_{i}$, and $w_{i}$ denote the constant-pressure specific heat capacity, the specific enthalpy, and the mass rate of production, respectively, of species $i$. The transport coefficients, thermodynamic properties, and rates of production are defined as usual; their functional form is specified below. The viscous 
terms $U$ and $V$ appearing in the $x$ and $y$ momentum equation, respectively, are given by

$$
\begin{aligned}
U= & \frac{\mu}{3} \frac{\partial(\nabla \cdot \boldsymbol{v})}{\partial x}+\frac{\partial \mu}{\partial x}\left(\frac{\partial u}{\partial x}-\frac{2}{3}(\nabla \cdot \boldsymbol{v})\right) \\
& +\frac{\partial \mu}{\partial y} \frac{\partial v}{\partial x}, \\
V= & \frac{\mu}{3} \frac{\partial(\nabla \cdot \boldsymbol{v})}{\partial y}+\frac{\partial \mu}{\partial y}\left(\frac{\partial v}{\partial y}-\frac{2}{3}(\nabla \cdot \boldsymbol{v})\right) \\
& +\frac{\partial \mu}{\partial x} \frac{\partial u}{\partial y},
\end{aligned}
$$

with the divergence of the velocity field given by

$$
\nabla \cdot \boldsymbol{v}=\frac{\partial u}{\partial x}+\frac{\partial v}{\partial y}+A
$$

The term $H$ appearing in Eq. 9 involves spatial derivatives of $c_{p}$ and, subsequently, will be neglected. In deriving Eqs. 11a and 11b use has been made of the fact that $\mu$ is independent of $z$. We note that in the special case of constant density and constant viscosity, we have $U=V$ $=0$.

The system of governing equations $5-10$ is closed through the ideal-gas equaiton of state

$$
\frac{p_{0}}{\rho}=R^{0} T \sum_{i=1}^{N}\left(\frac{Y_{i}}{W_{i}}\right),
$$

where $R^{0}$ denotes the universal gas constant and $W_{i}$ the molecular weight (= molar mass) of species $i$. Note that in Eq. 13 the pressure is taken as constant, an approximation which is consistent with the assumption of low-Machnumber flow.

The solution of the problem defined by Eqs. 5-10 and 13 requires appropriate boundary conditions to be imposed. At the fuel and oxidizer side, $y=+\infty$ and $y=-\infty$, respectively, the temperature and composition of either stream is specified. The $y$ velocity component $v$ in the fuel and oxidizer stream is $v_{F}=$ $-A_{F} y+a(x)$ and $v_{O}=-A_{O} y+b(x)$, respectively; here $a(x)$ and $b(x)$ are unknown functions characterizing the flame's displacement due to the heat release; in general, $a(x)$ and $b(x)$ must be determined as part of the solution of the problem. Note that the inviscid flow outside the stagnation region imposes the condition $\rho_{O} A_{O}^{2}=\rho_{F} A_{F}^{2}$. Sufficiently far upstream ahead of the flame, and sufficiently far downstream behind the flame, the derivatives with respect to $x$ of all dependent variables become negligibly small. Thus, as boundary conditions at $x=-\infty$ we impose a frozen similarity solution, which satisfies both the governing equations and the boundary conditions at $y= \pm \infty$ with $\partial / \partial x=0$. At $x=+\infty$ we require zero $x$ derivatives for all dependent variables.

\subsection{Simplifications}

Subsequently we assume equal and constant specific heat capacities $c_{p i}\left(=c_{p}\right)$ and equal diffusion coefficients $D_{i}(=D)$ for all species, and constant Prandtl and Lewis numbers of unity. Chemistry is assumed to occur via the global one-step reaction

$\nu_{F} F+\nu_{O} O \rightarrow \nu_{P} P$,

whose heat of reaction is $q=\nu_{P} W_{P} h_{P}-$ $\nu_{F} W_{F} h_{F}-\nu_{O} W_{O} h_{O}$; here the $\nu_{i}$ and $h_{i}$ denote the stoichiometric coefficient and (constant) specific enthalpy, respectively, of species $i, i=$ $F, O$, or $P$. The rate of reaction 14 is assumed to be of the Arrhenius form

$\omega=\left(\frac{\rho Y_{F}}{W_{F}}\right)\left(\frac{\rho Y_{O}}{W_{O}}\right) B \exp \left(-E / R^{0} T\right)$,

where the symbols have their usual meaning. With these specifications the energy and species conservation Eqs. 9 and 10 reduce to

$$
L(T ; \rho D)=-\frac{q}{c_{p}} \omega
$$

and

$L\left(Y_{F} ; \rho D\right)=-\nu_{F} W_{F} \omega$,

$L\left(Y_{O} ; \rho D\right)=-\nu_{O} W_{O} \omega$,

$L\left(Y_{p} ; \rho D\right)=\nu_{P} W_{P} \omega$.

Note that the mass fraction of an inert species $I$, which may be present in the system, is given by $Y_{I}=1-Y_{F}-Y_{O}-Y_{P}$. 
It will be advantageous to introduce a conserved scalar $Z$,

$Z=\frac{\frac{Y_{F}}{Y_{F, F}} s+\left(1-\frac{Y_{O}}{Y_{O, o}}\right)}{s+1}$,

where

$s=\frac{\nu_{O} W_{o} / Y_{O, o}}{\nu_{F} W_{F} / Y_{F, F}}$

is the air-fuel equivalence ratio. The conserved scalar obeys the governing equation

$L(Z ; \rho D)=0$,

which must be solved subject to the upstream boundary condition

$$
\begin{aligned}
Z & =\frac{I(y)}{I(\infty)}, \\
I(y) & =\int_{-\infty}^{y} \exp \left(\int_{-\infty}^{y^{\prime}} \frac{\rho v}{\rho D} d y^{\prime \prime}\right) d y^{\prime},
\end{aligned}
$$

and subject to $Z \rightarrow 1$ as $y \rightarrow+\infty, Z \rightarrow 0$ as $y \rightarrow-\infty$ and $\partial Z / \partial x=0$ as $x \rightarrow \pm \infty$.

We may combine Eqs. 16 and 20 to obtain

$$
\begin{aligned}
& L\left(Y_{1}\right)=-\nu_{F} W_{F} \omega, \\
& L\left(Y_{2}\right)=-\nu_{O} W_{O} \omega,
\end{aligned}
$$

where

$$
\begin{gathered}
Y_{1} \equiv \frac{\nu_{F} W_{F}}{q / c_{p}} T+\phi_{F} Z+\varphi_{F}, \\
Y_{2} \equiv \frac{\nu_{O} W_{O}}{q / c_{p}} T+\phi_{O} Z+\varphi_{O}
\end{gathered}
$$

are coupling functions $\phi_{O}, \phi_{F}, \varphi_{O}$, and $\varphi_{F}$ are constants to be determined. By virtue of Eqs. $21 \mathrm{a}$ and $21 \mathrm{~b}$, and upon requiring that at the boundaries $Y_{1}$ and $Y_{2}$ be identical to the fuel and the oxidizer mass fraction, repectively, we obtain

$$
\begin{aligned}
Y_{F}= & \frac{\nu_{F} W_{F}}{q / c_{p}}\left[T+Z\left(T_{O}-T_{F}\right)-T_{O}\right] \\
& +Z\left(Y_{F, F}-Y_{F, O}\right)+Y_{F, O} \\
Y_{O}= & \frac{\nu_{O} W_{O}}{q / c_{p}}\left[T+Z\left(T_{O}-T_{F}\right)-T_{O}\right] \\
& +Z\left(Y_{O, F}-Y_{O, O}\right)+Y_{O, O} .
\end{aligned}
$$

Upon eliminating $Z$ from Eqs. 23a and 23b, the coupling function

$\phi_{1} \frac{Y_{F}-Y_{F, O}}{\nu_{F} W_{F}}-\phi_{2} \frac{Y_{O}-Y_{O, o}}{\nu_{O} W_{O}}-\frac{T-T_{O}}{q / c_{p}}=0$

is obtained, where

$$
\begin{aligned}
\phi_{1}= & \left(\frac{T_{O}-T_{F}}{q / c_{p}}-\frac{Y_{O, O}-Y_{O, F}}{\nu_{O} W_{O}}\right) / \\
& \left(\frac{Y_{O, F}-Y_{O, O}}{\nu_{O} W_{O}}-\frac{Y_{F, F}-Y_{F, O}}{\nu_{F} W_{F}}\right) \\
\phi_{2}= & \left(\frac{T_{O}-T_{F}}{q / c_{p}}+\frac{Y_{F, F}-Y_{F, O}}{\nu_{F} W_{F}}\right) / \\
& \left(\frac{Y_{O, F}-Y_{O, O}}{\nu_{O} W_{O}}-\frac{Y_{F, F}-Y_{F, O}}{\nu_{F} W_{F}}\right),
\end{aligned}
$$

Upon introducing the temperature $T_{f}$ of the trailing diffusion-flame sheet,

$T_{f}=T_{O}-\frac{q}{c_{p}}\left(\phi_{1} \frac{Y_{F, O}}{\nu_{F} W_{F}}-\phi_{2} \frac{Y_{O, O}}{\nu_{O} W_{O}}\right)$,

Eq. 24 can be written as

$$
\begin{aligned}
\phi_{1} \frac{Y_{F}-Y_{F, O}}{\nu_{F} W_{F}}-\phi_{2} \frac{Y_{O}-Y_{O, O}}{\nu_{O} W_{O}} \\
+\frac{T-T_{O}}{T_{f}-T_{O}}\left(\phi_{1} \frac{Y_{F, O}}{\nu_{F} W_{F}}-\phi_{2} \frac{Y_{O, O}}{\nu_{O} W_{O}}\right)=0 .
\end{aligned}
$$

\subsection{Nondimensionalization}

The problem is nondimensional by introducing the scaled variables

$$
\begin{aligned}
& \tilde{t}=t A_{O}, \quad \tilde{x}=x / \sqrt{D_{O} / A_{O}}, \\
& \tilde{y}=y / \sqrt{D_{O} / A_{O}},
\end{aligned}
$$

and

$$
\begin{aligned}
\tilde{A} & =A / A_{0}, \quad \tilde{u}=u / \sqrt{D_{O} A_{O}}, \\
\tilde{v} & =v / \sqrt{D_{O} A_{O}}, \quad \tilde{p}^{\prime}=p^{\prime} / \rho_{O} A_{O} D_{O}, \\
\tilde{\rho} & =\rho / \rho_{O}, \quad \tilde{Y}_{F}=Y_{F} / Y_{F, F}, \\
\tilde{Y}_{O} & =Y_{O} / Y_{O, O}, \quad \tilde{T}=\left(T-T_{O}\right) /\left(T_{f}-T_{O}\right) .
\end{aligned}
$$


In terms of these variables, the governing equations can be written as

$$
\begin{aligned}
\tilde{L}(1 ; 1) & =0 \\
\tilde{L}(\tilde{u} ; \tilde{\mu} \mathrm{Sc}) & =-\frac{\partial \tilde{p}^{\prime}}{\partial \tilde{x}}+\tilde{U} \mathrm{Sc}, \\
\tilde{L}(\tilde{\boldsymbol{v}} ; \tilde{\mu} \mathrm{Sc}) & =-\frac{\partial \tilde{p}^{\prime}}{\partial \tilde{y}}+\tilde{\rho} \tilde{y}+\tilde{V} \mathrm{Sc}, \\
\tilde{L}(\tilde{A} ; \tilde{\mu} \mathrm{Sc}) & =\tilde{\rho}\left(1-\tilde{A}^{2}\right), \\
\tilde{L}(\tilde{Y} ; \tilde{\rho} \tilde{D})= & -\delta \beta^{4 \tilde{\rho}^{2}} \tilde{Y}_{F} \tilde{Y}_{O} \exp (-\beta(1-\tilde{T}) / \\
& {[1-\alpha(1-\tilde{T})]), } \\
\tilde{L}(\tilde{Z} ; \tilde{\rho} \tilde{D})= & 0,
\end{aligned}
$$

where the operator $\tilde{L}$ is defined analogously to $L$, and where $\tilde{Z} \equiv Z$. The boundary conditions are nondimensionalized analogously. In Eqs. 30-35, the diffusion coefficient and dynamic viscosity have been nondimensionalized with their respective values at the oxidizer side,

$\mathrm{Sc}=\mu_{O} /\left(\rho_{O} D_{O}\right)$

is a Schmidt number,

$\beta=\frac{E}{R^{0} T_{f}} \frac{T_{f}-T_{O}}{T_{f}}$

is the Zel'dovich number, which is assumed to be large,

$\alpha=\frac{T_{f}-T_{O}}{T_{f}}$

a nondimensional heat-release parameter, and $\delta=\frac{\nu_{F} \rho_{O} Y_{O, O} B}{A_{O} W_{O}} \exp \left(-E / R^{0} T_{f}\right) / \beta^{4}$

a Damköhler number. The nondimensional temperature and density are given by

$\tilde{T}=1-\phi_{3} \tilde{Y}_{F}+\phi_{4} \tilde{Y}_{O}$,

$\tilde{\rho}=(1-\alpha) /[1-\alpha(1-\tilde{T})] ;$ the nondimensional oxidizer mass fraction is obtained from Eq. 18, viz.

$\tilde{Y}_{O}=1+s \tilde{Y}_{F}-(s+1) \tilde{Z}$.

The quantities $\phi_{3}$ and $\phi_{4}$ appearing in Eq. 40 are given by

$\phi_{3}=\frac{\phi_{1} Y_{F, F} /\left(\nu_{F} W_{F}\right)}{\phi_{1} Y_{F, O} /\left(\nu_{F} W_{F}\right)+\phi_{2} Y_{O, O} /\left(\nu_{O} W_{O}\right)}$,

$\phi_{4}=\frac{\phi_{2} Y_{O, o} /\left(\nu_{O} W_{O}\right)}{\phi_{1} Y_{F, o} /\left(\nu_{F} W_{F}\right)+\phi_{2} Y_{O, o} /\left(\nu_{O} W_{O}\right)}$.

\subsection{The Thermal-Diffusional Model}

We now turn to the limiting case of a thermaldiffusional model, that is, to the case in which $\alpha \rightarrow 0$. In this case we have $\bar{\rho}=1, \tilde{u}=\tilde{u}_{F}$, $\tilde{v}=-\tilde{y}$, and $\bar{A}=1$. Assuming $\tilde{D}=1$, the set of governing equations reduces to

$$
\begin{aligned}
\tilde{u}_{F} \frac{\partial \tilde{Y}_{F}}{\partial \tilde{x}}-\tilde{y} \frac{\partial \tilde{Y}_{F}}{\partial \tilde{y}} & =\frac{\partial^{2} \tilde{Y}_{F}}{\partial \tilde{x}^{2}}+\frac{\partial^{2} \tilde{Y}_{F}}{\partial \tilde{y}^{2}} \\
& -\delta \beta^{4} \tilde{Y}_{F} \tilde{Y}_{O} e^{-\beta(1-\tilde{T})}, \\
\tilde{u}_{F} \frac{\partial \tilde{Z}}{\partial \tilde{x}}-\tilde{y} \frac{\partial \tilde{Z}}{\partial \tilde{y}} & =\frac{\partial^{2} \tilde{Z}}{\partial \tilde{x}^{2}}+\frac{\partial^{2} \tilde{Z}}{\partial \tilde{y}^{2}} \\
\tilde{T} & =1-\tilde{Y}_{F}-\tilde{Y}_{O}, \\
\tilde{Z} & =\frac{s \tilde{Y}_{F}+1-\tilde{Y}_{O}}{s+1},
\end{aligned}
$$

with the boundary conditions

$$
\begin{aligned}
\tilde{y} \rightarrow-\infty: \tilde{Y}_{F} & =\tilde{Z}=0, \\
\tilde{y} \rightarrow+\infty: \tilde{Y}_{F}= & \tilde{Z}=1, \\
\tilde{x} \rightarrow-\infty: \tilde{Z}= & \frac{1}{2} \frac{s+1}{s Y_{F, F}+1-Y_{O, O}} \\
& \times \operatorname{erfc}(\tilde{y} / \sqrt{2}) \text { and }
\end{aligned}
$$

$\frac{\partial \tilde{Z}}{\partial \tilde{x}}=\frac{\partial \tilde{Y}_{F}}{\partial \tilde{x}}=0$,

$\tilde{x} \rightarrow+\infty$ : zero $x$ gradients for all dependent variables.

Recall that the nondimensional flame speed $\tilde{u}_{F}$ is an eigenvalue that must be determined as 
part of the solution. The procedure to determine $\tilde{u}_{F}$ is described in subsubsection 3.5.1.

\subsection{NUMERICAL METHOD}

\subsubsection{Difference Equations}

For ease of notation, in this subsection the tilde is dropped from the nondimensional variables. Equations 43 and 44 are used in 41 and 42 to eliminate $Y_{O}$ and $T$. With respect to the space variables $x$ and $y$, the two strongly coupled equations 41 and 42 are discretized on a mesh $\mathbf{M}^{n}$ of grid points,

$\mathbf{M}^{n}=\left\{\left(x_{i}^{n}, y_{j}^{n}\right) ; i=1, \ldots, N_{x}^{n}, j=1, \ldots, N_{y}^{n}\right\}$.

Here the grid lines $x_{i}^{n}=$ constant, $i=$ $1, \ldots, N_{x}^{n}$, and $y_{j}^{n}=$ constant $, j=1, \ldots, N_{y}^{n}$, are distributed in a nonequidistant manner using the adaptive-gridding procedure described below. In Eq. 48 and below the superscript $n$ identifies quantities at time level $t^{n}$, $n=0,1,2, \ldots$; if possible without ambiguity, subsequently for ease of notation this superscript will be omitted. For the first-order derivatives central differences are adopted, for example, for the $x$ derivative of a scalar dependent variable $\phi$ at the grid point with the coordinates $\left(x_{i}, y_{j}\right)$, we write

$$
\begin{aligned}
\left(\frac{\partial \phi}{\partial x}\right)_{i, j} \approx & {\left[\frac{h_{i-1}}{h_{i}\left(h_{i}+h_{i-1}\right)} \phi_{i+1, j}\right.} \\
& +\frac{h_{i}-h_{i-1}}{h_{i} h_{i-1}} \phi_{i, j} \\
& \left.-\frac{h_{i}}{h_{i-1}\left(h_{i}+h_{i-1}\right)} \phi_{i-1, j}\right],
\end{aligned}
$$

where $h_{i}=x_{i}-x_{i-1}, i=2, \ldots, N_{x}$. The second-order derivatives at grid point $\left(x_{i}, y_{j}\right)$, for example, in the $x$ direction, are approximated by

$$
\begin{aligned}
\left(\frac{\partial^{2} \phi}{\partial x^{2}}\right)_{i, j} \approx & \frac{2}{h_{i}+h_{i+1}} \\
& \times\left[\frac{\phi_{i+1, j}-\phi_{i, j}}{h_{i}}-\frac{\phi_{i, j}-\phi_{i-1, j}}{h_{i-1}}\right] .
\end{aligned}
$$

To bring the initially guessed solution of the problem into the domain of convergence of Newton's method applied to steady-state version of the governing equations, terms involving time derivatives are added to the governing equations. Since we are interested only in steady-state solutions, temporal accuracy of the solution is unimportant and, therefore, the time derivatives are approximated by simple backward-Euler finite-differences. A fully implicit formulation is employed in order to successfully cope with the stiffness of system 41-44 that arises through the chemical source term in Eq. 41.

We now describe the procedure adopted herein to determine the burning-rate eigenvalue $u_{F}$ for a given set of parameters $\beta, \delta$, and $s$. We first note that since the solution to the governing equations is translational invariant, the condition

$T=T_{\mathrm{fix}} \quad$ at $i=i_{\mathrm{fix}}, \quad j=j_{\mathrm{fix}}$

is imposed in order to "anchor" the flame in the computational domain. Secondly we note that the solution to a problem is independent of the value selected for the temperature $T_{\text {fix }}$ and of the location at which the temperature, and hence the flame, is fixed. Herein $T_{\text {fix }}=0.5$ has been selected. The burning-rate eigenvalue is treated as an additional dependent variable that obeys the differential equations

$\frac{\partial u_{F}}{\partial x}=\frac{\partial u_{F}}{\partial y}=0$.

Equations 51 are discretized according to

$$
\begin{aligned}
& \left(\frac{\partial u}{\partial x}\right)_{i, j}=\left\{\begin{array}{c}
\left(u_{i+1, j}\right) /\left(x_{i+1, j}-x_{i, j}\right) \\
\text { for } i<i_{\mathrm{fix}}, 1 \leq j \leq N_{y}, \\
\left(u_{i, j}-u_{i-1, j}\right) /\left(x_{i, j}-x_{i-1, j}\right) \\
\text { for } i>i_{\mathrm{fix}}, 1 \leq j \leq N_{y},
\end{array}\right. \\
& \left(\frac{\partial u}{\partial y}\right)_{i, j}=\left\{\begin{array}{c}
\left(u_{i, j+1}-u_{i, j}\right) /\left(y_{j+1, j}-y_{i, j}\right) \\
\text { for } j<j_{\mathrm{fix}}, i=i_{\mathrm{fix}}, \\
\left(\begin{array}{c}
\left.u_{i, j}-u_{i, j-1}\right) /\left(y_{i, j}-y_{i, j-1}\right) \\
\text { for } j>j_{\mathrm{fix}}, i=i_{\mathrm{fix}},
\end{array}\right.
\end{array}\right.
\end{aligned}
$$

where for ease of notation we have dropped the subscript $F$ from $u_{F}$. If Newton's method 
is used to solve the governing equations in terms of the dependent variables $Y_{F}, Z$, and $u_{F}$, the discretization of these variables as described above leads to a Jacobian matrix with a block-pentadiagonal structure.

\subsubsection{Newton's Method}

Subsequently the symbols $\mathbf{U}$ and $\mathbf{F}$ will be used to denote the $N_{x} \times N_{y} \times N$-vectors that result from the spatial discretization of the system of governing equations. (Herein we have $N=3$ partial differential equations.) For the solution of the time-dependent problem at each time step, or for the steady-state problem, Newton's method is applied to the system of nonlinear equations, $\mathbf{F}(\mathbf{U})$, which results from the discretization of the governing equations. Note that both $\mathbf{U}$ and $\mathbf{F}$ depend on the particular time level $n$ under consideration. Thus, the linear system

$$
\begin{array}{r}
J\left(\mathbf{U}^{k}\right)\left(\mathbf{U}^{k+1}-\mathbf{U}^{k}\right)=-\omega_{k} \mathbf{F}\left(\mathbf{U}^{k}\right), \\
\quad k=0,1, \ldots,
\end{array}
$$

is solved, where $\mathbf{U}^{k}$ denotes the solution after $k$ Newtonian iterations, and $\omega_{k}$ and $J\left(\mathbf{U}^{k}\right)$ are the damping parameter [10] and the Jacobian matrix, respectively, based on $\mathbf{U}^{k}$. The Jacobian, which is generated numerically, is reevaluated only periodically [11].

The solution of the system of equations at time level $n$ depends on the solution at level $n-1$ taken at the grid points of mesh $\mathbf{M}^{n}$. Since in general the grids at levels $n-1$ and $n$ are not the same, the solution obtained at level $n-1$ on grid $\mathbf{M}^{n-1}$ must be interpolated onto grid $\mathbf{M}^{n}$, which, regardless of the interpolation procedure, introduces an additional spatial discretization error into the algorithm. We use piecewise monotonic cubic Hermite interpolation [12].

\subsubsection{Adaptive Selection of Grid Points}

The procedures and criteria for the adaptive selection of grid points are of critical importance to the efficiency of the algorithms that are used for the solution of combustion problems. In particular, strategies are required that place the grid points where they are needed, that is, in regions where the dependent variables exhibit steep gradients and/or strong curvature, in order to bound the local spacediscretization error. In generalization of procedures outlined previously for the adaptive computation of steady one-dimensional combustion problems [13], for any fixed time level $n$ we equidistribute the mesh $\mathbf{M}^{n}$ on intervals $h_{i}^{n}=x_{i}^{n}-x_{i-1}^{n}, k_{j}^{n}=y_{j}^{n}-y_{j-1}^{n}$ with respect to a non-negative weight functions $W_{x}^{n}, W_{y}^{n}$ and constants $C_{x}^{n}, C_{y}^{n}$. For instance, for the $x$ direction $W_{x}^{n}$ is selected such that

$$
\int_{h_{i}^{n}} W_{x}^{n} d x=C_{x}^{n}, \quad i=2, \ldots, N_{x}^{n}
$$

Specifically, the weight function $W_{x}^{n}$ is chosen as

$$
\begin{aligned}
W_{x}^{n} & =\max _{1 \leq k \leq 2 N+1} W_{x, k}^{n}, \\
W_{x, k}^{n}= & \frac{\left|\partial U_{k} / \partial x\right|}{g_{x}^{n}\left|\max U_{k}-\min U_{k}\right|}, \\
1 \leq k \leq N_{x}^{n} & (55 \mathrm{~b}) \\
W_{x, N+k}^{n}= & \frac{\left|\partial^{2} U_{k} / \partial x^{2}\right|}{c_{x}^{n}\left|\max \left(\partial U_{k} / \partial x\right)-\min \left(\partial U_{k} / \partial x\right)\right|}, \\
W_{x, 2 N+1}^{n}= & d^{n} .
\end{aligned}
$$

In Eqs. 55b and 55c "min" and "max" stand for the minimum and maximum value in the domain of integration of the respective quantity, and $g_{x}^{n}$ and $c_{x}^{n}$ are positive scaling factors; their numerical values are less than unity if in Eq. $54 C_{x}^{n}=1$ is employed. In Eq. 55 d, $d^{n}$ is a positive constant that represents the maximum size of any interval $h_{i}$. To prevent the size of adjacent mesh intervals from varying too rapidly, we require that at any time level $n$ the mesh be locally bounded, viz.,

$R_{x}^{-1} \leq h_{i}^{n} / h_{i-1}^{n} \leq R_{x}$,

where $R_{x}$ is a constant greater than one. The equidistribution procedure with respect to the $y$ direction is performed analogously. 
The adaptive-gridding procedure to be carried out at each time level $n$ essentially consists of seven steps, viz.,

step 1 Select an initial grid $\mathbf{M}^{0}$ and specify initial profiles on $\mathbf{M}^{0}$

step 2 Initialize $n=k=0$ and

$$
\mathbf{M}_{\text {converged }}=\mathbf{M}^{0}
$$

step 3 If $n=n_{\text {max }}$ : Stop

step 4 Set $n=n+1$ and then

$$
\mathbf{M}_{k}^{n}=\mathbf{M}_{\text {converged }}
$$

step 5 Solve (1) on $\mathbf{M}_{k}^{n}$

step 6 Calculate $\mathbf{M}_{k+1}^{n}$

step 7 If $\mathbf{M}_{k+1}^{n} \mathbf{K}$ equals $\mathbf{M}_{k}^{n}$ then

Set $\mathbf{M}_{\text {converged }}=\mathbf{M}_{k}^{n}$

Set $k=0$

Go to step 4

Else

Interpolate the solution to (1)

onto $\mathbf{M}_{k+1}^{n}$

Set $k=k+1$

Go to step 5

\section{End If}

Equation 57 calls for some comments. Firstly, the "Stop" in step 3 may be replaced by any other suitable command. For instance, if the time-dependent integration is carried out with the aim of bringing the initial profiles into the domain of convergence of Newton's method applied to the steady version of Eqs. 41-44, then the "Stop" may be replaced by "Try to solve the steady-state problem." Secondly, in step 7 the meaning of " $\mathbf{M}_{k+1}^{n}$ equals $\mathbf{M}_{k}^{n}$ " needs to be specified. In order to determine whether two consecutive grids are "equal," we employ two different criteria. One, called grid convergence, is that for two consecutive grids $k-1$ and $k$ the number of grid lines in $x$ and $y$ direction, respectively, is the same, say $N_{x, k}^{n}$ and $N_{y, k}^{n}$, and that in addition

$$
\begin{aligned}
& \frac{1}{N_{x, k}^{n}} \sum_{i=1}^{N_{x, k}^{n}}\left(x_{i, k}^{n}-x_{i, k-1}^{n}\right)^{2} \leq \epsilon, \\
& \frac{1}{N_{y, k}^{n}} \sum_{j=1}^{N_{y, k}^{n}}\left(y_{j, k}^{n}-y_{j, k-1}^{n}\right)^{2} \leq \epsilon,
\end{aligned}
$$

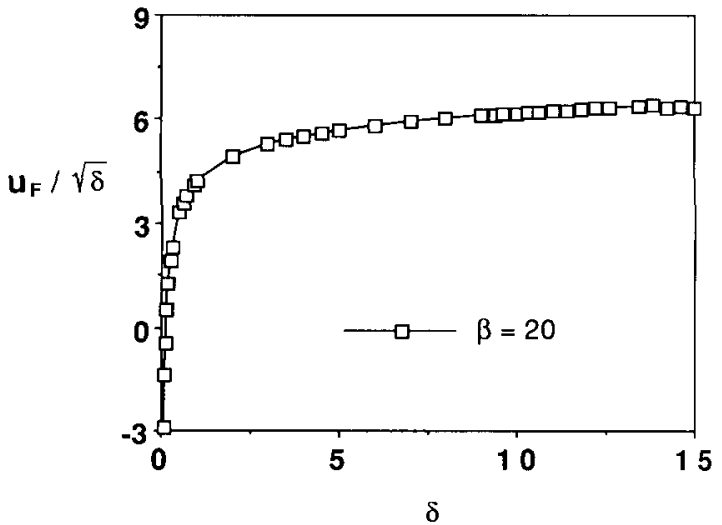

Fig. 5. Computed ratio of the burning-rate eigenvalue $\tilde{u}_{F}$ to the Damköhler number $\delta, \tilde{u}_{F} / \sqrt{\delta}$, as a function of $\delta$, for $\beta=20$.

where $\epsilon$ is small positive constant. The other, called alternating grid convergence, is that repeatedly alternating insertion and removal of the same number (typically 1 or 2 ) of grid lines occurs.

\subsection{Numerical Results and Discussion}

The results presented and discussed below were obtained for $s=1$, where $s$ is the air-fuel equivalence ratio defined in Eq. 19. Thus, all numerical results presented are of triple flames which are symmetric about the $\tilde{x}$-axis. Shown in Fig. 5 is the ratio of the burning-rate eigenvalue $\tilde{u}_{F}$ to the Damköhler number $\delta, \tilde{u}_{F} / \sqrt{\delta}$,

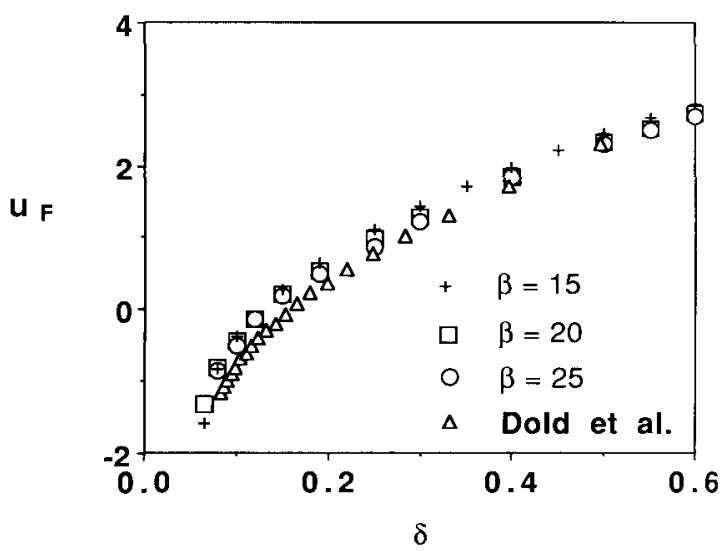

Fig. 6. Computed propagation velocity $\tilde{u}_{F}$ as a function of the Damköhler number $\delta$ for small values of $\delta$ as predicted by the present computations for $\beta=15,20$, and 25 and the asymptotic prediction taken from Ref. 6 . 
as a function of $\delta$ for $\beta=20$. Note that according to $\mathrm{Eq} .39, \delta$ is proportional to the reciprocal of the strainrate $A_{O}$. It is seen from Fig. 5, that (i) for low values of $\delta$ the ratio $\bar{u}_{F} / \sqrt{\delta}$ and hence the propagation velocity is negative and (ii) for large values of $\delta$ the ratio $\tilde{u}_{F} / \sqrt{\delta}$ approaches a constant value. These findings are in accordance with prediction by asymptotic analysis [6]. Shown in Fig. 6 is the propagation velocity $\tilde{u}_{F}$ as a function of $\delta$ for small values of $\delta$ as predicted by the present computations for $\beta=15,20$, and 25 and the asymptotic predictions taken from Ref. 6. It is seen that the agreement between the numerical predictions and the result of the asymptotic analysis is good.

Shown in Figs. $7 a-7 c$ are contour plots in the $\tilde{x}-\tilde{y}$ plane of nondimensional coordinates of the nondimensional reaction rate

$\Omega \equiv \delta \beta^{4} \tilde{Y}_{F} \tilde{Y}_{O} e^{-\beta(1-\tilde{T})}$,

the normalized temperature $\tilde{T}$ and fuel mass fraction $\bar{Y}_{F}$ for a triple flame with $\beta=20$ and

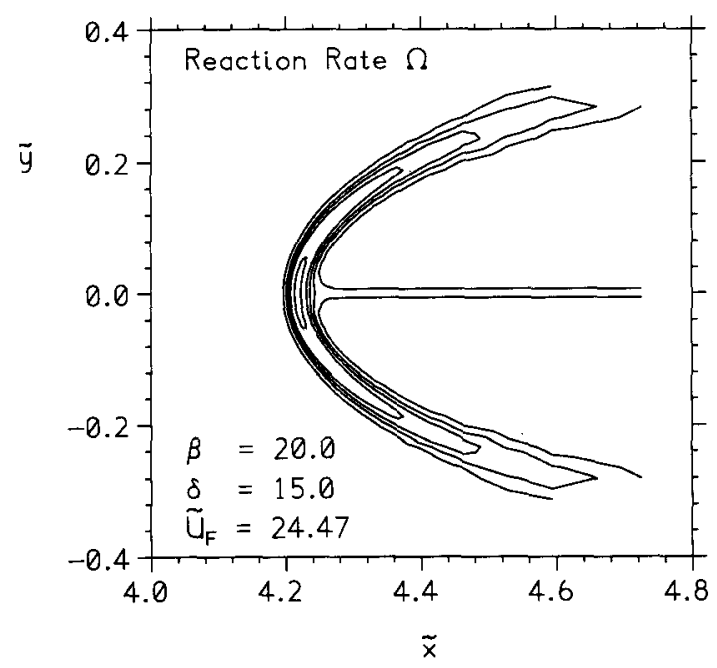

(a)

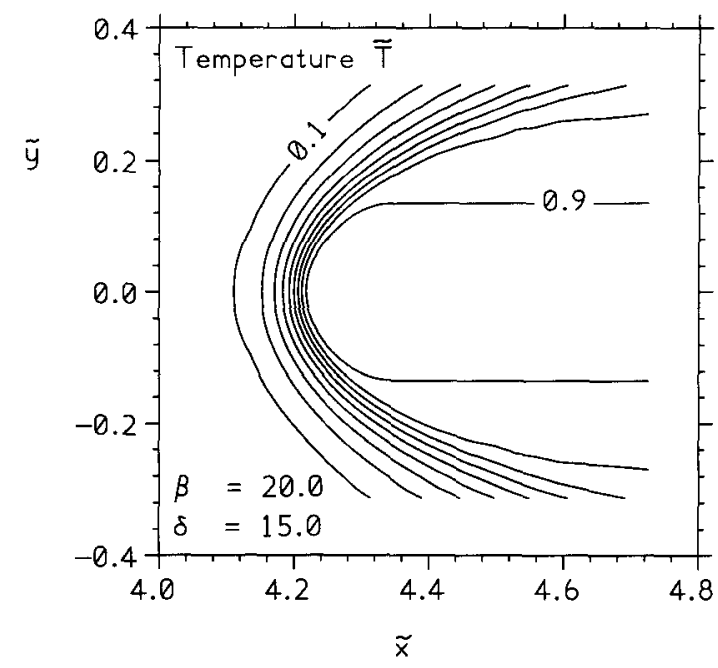

(b)

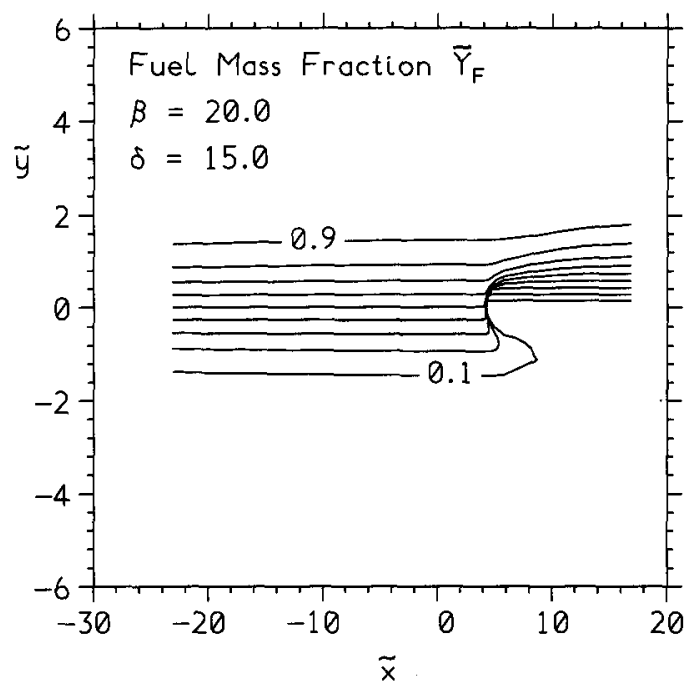

(c)

Fig. 7. Contour plots in the $\bar{x}-\tilde{y}$ plane of nondimensional coordinates of (a) the nondimensional reaction rate $\Omega$, (b) the nondimensional temperature $\tilde{T}$, and (c) the normalized fuel mass fraction $\tilde{Y}_{F}$, all for a triple flame with $\beta=20$ and $\delta=15$. 
$\delta=15$. In Fig. 7a the triple-flame structure is clearly visible: the two backward bending premixed wings joint in the triple point with the trailing diffusion flame. The triple-flame structure is less easily identified from the contour plot of the nondimensional temperature, Fig. 7b. It manifests itself, however, in the contour plot of the normalised fuel mass fraction, Fig. 7c. In the latter figure, the inert mixing ahead of the flame is represented by the diffusion of fuel to the oxidizer side; the back bending contours at $\tilde{x} \approx 4.31$ clearly indicate that there combustion takes place in the premixed mode. Finally, the trailing, parallel contour lines of fuel mass fraction show that the fuel goes to depletion in the thin, trailing diffusion flame. For the flame of Fig. 7, shown in Fig. 8 are sections of the nondimensional reaction rate $\Omega$ in the $\tilde{y}$ direction at axial locations $\tilde{x}=4.28$, $4.31,4.43$, and 4.95 . It is seen that for $\tilde{x}=4.28$ there is just a single maximum of $\Omega$ indicating the location of the triple point. For increasing values of $\tilde{x}, \Omega$ has two maxima, each maximum indicating one of the premixed flame wings; the local minimum of $\Omega$ between the two maxima represents the reaction rate in the diffusion-flame wing. It is seen from Fig. 8 that the reaction rate attains its highest value in the triple point and decreases slightly towards the trailing diffusion flame. Furthermore it can be seen that, as is to be expected on physical grounds, the reaction rate decreases in the triple flame with increasing values $\tilde{x}$.

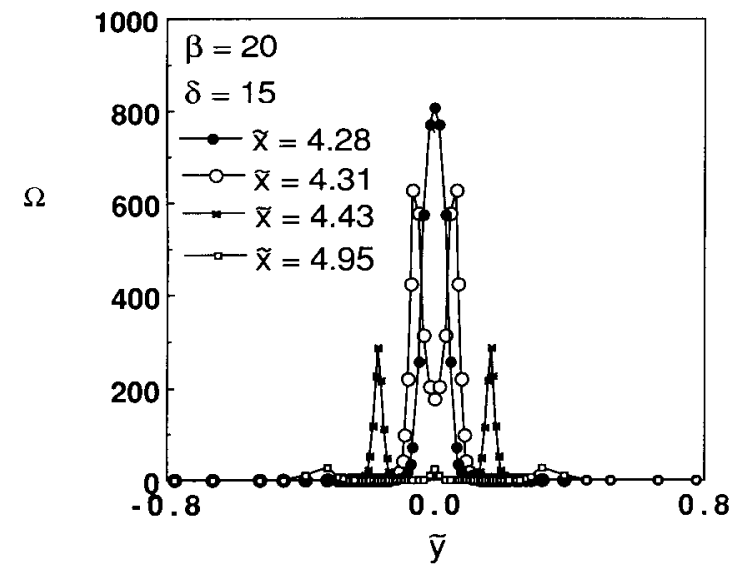

Fig. 8. Sections of the nondimensional reaction rate $\Omega$ in the $\vec{y}$ direction at axial locations $\tilde{x}=4.28,4.31,4.43$, and 4.95 for a triple flame with $\beta=20$ and $\delta=15$.
Figures $9 \mathrm{a}-9 \mathrm{c}$ illustrate the change in flame structure in terms of the nondimensional reaction rate $\Omega$ with increasing rate of strain, that is, with decreasing value of the Damköhler number. The numerical values for $\delta$ and $\bar{u}_{F}$ are: for Fig. 9a: $\delta=1.1$ and $\tilde{u}_{F}=4.73$, for Fig. $9 \mathrm{~b}: \delta=0.127$ and $\tilde{u}_{F}=3.64 \times 10^{-2}$, for Fig. 9c: $\delta=0.06$ and $\tilde{u}_{F}=-2.174$; in all three figures the numerical value for $\beta$ is 15 . The sequence of pictures shown in Figs. $9 a-9 c$ clearly shows that with increasing rate of strain the structure of the triple flame evolves towards the structure of an ordinary diffusion flame; it also shows that the transition in structure occurs at strain rates for which the propagation velocity of the flame is approximately zero. We note that with decreasing Damköhler number the nondimensional width of the premixed flame wings remains approximately constant, whereas the diffusion flame becomes thicker until for $\tilde{u}_{F} \approx 0$ the premixed wings and the tip of the diffusion flame merge. It should be noted that flame spread in negative direction is not a physical contradiction because the fuel and oxidizer required to sustain the flame are continuously supplied from the two sides of the mixing layer.

\section{CONCLUSIONS}

In the present paper we have achieved the following. Firstly, a burner design has been presented for the stabilization of triple flames in coflowing streams. First experimental results obtained with this burner were presented that lead to the conclusion that the burner is well suited for the experimental investigation of triple flames.

Secondly, a similarity formulation has been developed describing diffusion flame spread in strained, counterflowing streams. Here numerical, self-adaptive methods have been developed that are capable of solving the truly twodimensional governing equations. Using these methods, for the limiting case of the thermaldiffusional model the structure and propagation velocity of the triple flame have been computed for various values of the strainrate. In the computations it has been found that at large Damköhler numbers, that is, low rates of strain, the flame structure is indeed that of a 


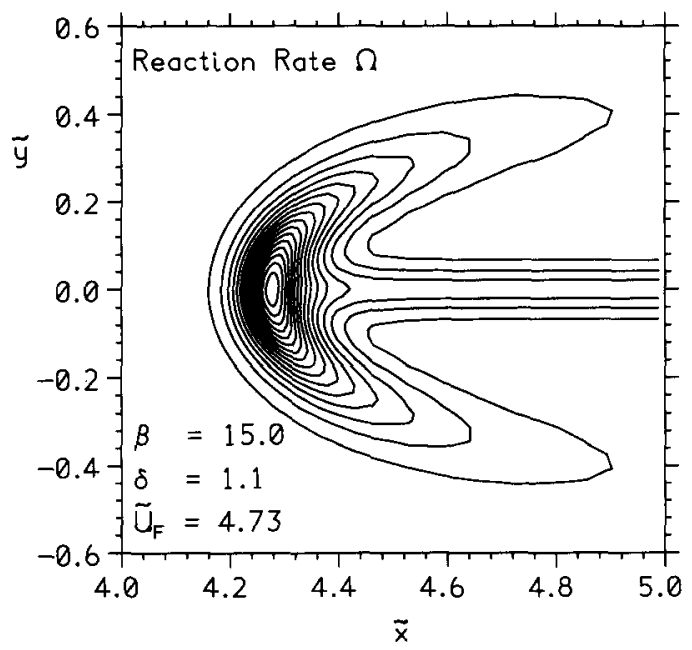

(a)

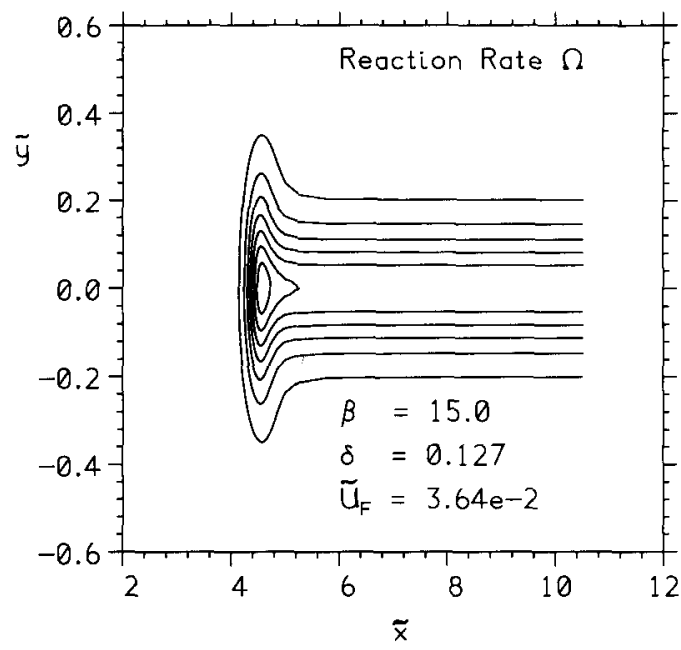

(b)

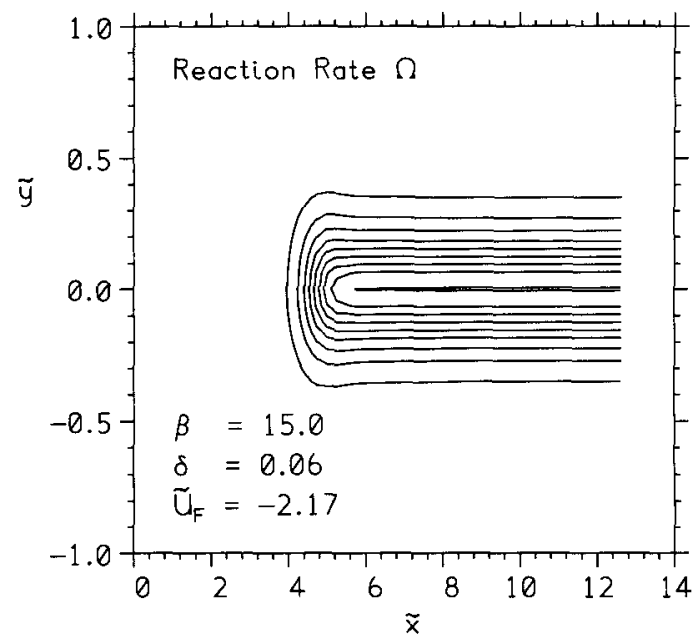

(c)

Fig. 9. Contour plots in the $\tilde{x}-\tilde{y}$ plane of nondimensional coordinates of the nondimensional reaction rate $\Omega$ for a flame with $\beta=15$ and (a) $\delta=1.1$, (b) $\delta=1.27$, (c) $\delta=0.06$.

triple flame propagating with a relatively large, positive velocity. With decreasing rates of strain, the propagation velocity decreases and the flame structure evolves towards that of an ordinary diffusion flame. For sufficiently high rates of strain the propagation velocity of the diffusion flame becomes negative.

The present study was supported by the Commission of the European Communities within the frame of the JOULE Programme, by the Swedish National Board for Technical Development, and by the Joint Research Committee of European automobile manufacturers (Fiat, Peugeot $S A$, Renault, Volkswagen, and Volvo) within the IDEA Programme. It was also supported by the Science and Engineering Research Council, UK, grant number $G R / F$ 25280, and by NATO, grant number 0101/89. One of the authors $(B R)$ would like to thank J. W. Dold for interesting and helpful discussions.

\section{REFERENCES}

1. Phillips, H., Tenth Symposium (International) on Combustion, The Combustion Institute, Pittsburgh, 1965, pp. $1277-1283$. 
2. Liñán, A., and Crespo, A., Combust. Sci. Technol. 14:95-117 (1976).

3. Dold, J. W., Prog. Astronaut. Aeronaut. 240-248 (1988).

4. Dold, J. W., Combust. Flame 76:71-88 (1989).

5. Dold, J. W., Paper presented at the Third International Seminar on Flame Structure, September 1989.

6. Dold, J. W., Hartley, L. J., and Green, D., in Dynamical Issues in Combustion Theory, IMA Volumes in Mathematics and its Applications (P. C. Fife, A. Liñán, and F. A. Williams, Eds.), Springer, New York, 1990.

7. Buckmaster, J., and Matalon, M., Twenty-Second Symposium (International) on Combustion, The Combustion Institute, Pittsburgh, pp. 1527-1535.
8. Wichman, I. S., Combust. Sci. Technol. 64:295-313 (1989).

9. Ramanujam, S., and T'ien, J. S., Paper presented at the 2nd ASME-JSME Thermal Engineering Joint Conference, March 22-27, 1987.

10. Deuflhard, P., Numer. Math. $22: 289$ (1974).

11. Smooke, M. D., J. Opt. Theory Appl. 39:489 (1983).

12. Fritsch, F. N., and Carlson, J., SLAM J. Numer. Anal. 17:238-246 (1980).

13. Giovangigli, V., and Smooke, M. D., Combust. Sci. Technol. 53:23 (1987).

Received 13 August 1991; revised 10 May 1993 